\title{
An Improved Optimal Slip Ratio Prediction considering Tyre Inflation Pressure Changes
}

\author{
Guoxing Li, ${ }^{1,2}$ Tie Wang, ${ }^{1}$ Ruiliang Zhang, ${ }^{1}$ Fengshou Gu, ${ }^{1,2}$ and Jinxian Shen ${ }^{1}$ \\ ${ }^{1}$ Department of Vehicle Engineering, Taiyuan University of Technology, Taiyuan 030024, China \\ ${ }^{2}$ Centre for Efficiency and Performance Engineering, University of Huddersfield, Huddersfield HD1 3DH, UK \\ Correspondence should be addressed to Tie Wang; wangtie57@163.com
}

Received 7 August 2015; Revised 2 November 2015; Accepted 10 November 2015

Academic Editor: Petko Petkov

Copyright (c) 2015 Guoxing Li et al. This is an open access article distributed under the Creative Commons Attribution License, which permits unrestricted use, distribution, and reproduction in any medium, provided the original work is properly cited.

\begin{abstract}
The prediction of optimal slip ratio is crucial to vehicle control systems. Many studies have verified there is a definitive impact of tyre pressure change on the optimal slip ratio. However, the existing method of optimal slip ratio prediction has not taken into account the influence of tyre pressure changes. By introducing a second-order factor, an improved optimal slip ratio prediction considering tyre inflation pressure is proposed in this paper. In order to verify and evaluate the performance of the improved prediction, a cosimulation platform is developed by using MATLAB/Simulink and CarSim software packages, achieving a comprehensive simulation study of vehicle braking performance cooperated with an ABS controller. The simulation results show that the braking distances and braking time under different tyre pressures and initial braking speeds are effectively shortened with the improved prediction of optimal slip ratio. When the tyre pressure is slightly lower than the nominal pressure, the difference of braking performances between original optimal slip ratio and improved optimal slip ratio is the most obvious.
\end{abstract}

\section{Introduction}

The longitudinal motion of a vehicle is governed by the forces generated between the tyres and the road surface. Therefore, acquiring enough tyre friction is crucial to enhance vehicle dynamics. According to the friction principle, the magnitude of frictional force depends on two factors: normal pressure and friction coefficient. However, the relationship between the longitudinal friction coefficient and wheel slip ratio is complex. In the premise of constant normal pressure, when the wheel slip ratio is small, longitudinal force linearly increases with slip ratio. With further increase of the slip ratio, longitudinal force increases and then decreases nonlinearly. When the longitudinal force reaches a maximum value, the corresponding slip ratio is called optimal slip ratio.

Antilock braking system (ABS) is an automobile safety system that allows the vehicle wheels to maintain tractive contact with the road surface according to driver inputs during the braking process, preventing the wheels from both locking up and uncontrolled skidding. The logic threshold control method is widely applied in commercial ABS products [1]. As an experience based control method, in the control process, wheel slip ratio is not maintained in optimal slip ratio but fluctuated near it which cannot acquire the best braking effect. Meanwhile, if slip ratio is considered as the control target, ABS controller can maintain the practical slip ratio near the optimal slip ratio all the time during the braking process, so that vehicle controllability and stability are optimized and maximized. That is considered as the ideal braking method. The research on ABS control, aiming at optimal slip ratio, has been carried out for many years. Most researchers put the emphasis on control strategy optimization and development of control methods based on the control theory of self-turning PID, fuzzy PID, artificial neural network, and so on [2-4]. Other researchers focused on identification of road surface and optimal slip ratio [5-7].

Up to now, most studies about optimal slip ratio control are based on an optimal slip ratio estimation expression, proposed by Liu [8] and Bian [9], which gives a quantification description of influence on optimal slip ratio due to changes of road adhesion coefficient, vehicle velocity, and tyre slip angle. The optimal slip ratio expression proposed by Liu et al. is mainly developed from the tyre Magic Formula, proposed by Pacejka [10], using regression analysis. In the 
early time, the influence of tyre inflation pressure changes was not considered into Magic Formula based on which the influence has not been put into existing optimal slip ratio expression.

However, it is indicated in [11-13] that tyre inflation pressure changes can directly influence the relationship between tyre longitudinal force and slip ratio. That is, the value of optimal slip ratio is influenced by tyre inflation pressure changes.

Based on the existing optimal slip ratio expression and an improved Magic Formula model [14], a simulation is launched to make a study on relationship between tyre inflation pressure changes and tyre slip ratio. A second-order factor, representing the influence of tyre inflation pressure changes on the value of optimal slip ratio, is acquired. In order to verify and evaluate the improved optimal slip ratio, an ABS controller with optimal slip ratio as the control target is established. Vehicle braking processes before and after improvement are simulated and compared.

The paper is structured as follows. Section 2 gives a simple introduction of tyre dynamic principle and optimal slip ratio. Section 3 describes the proposed improved optimal slip ratio considering tyre inflation pressures. Section 4 describes the modelling of cosimulation system which consists of a fuzzy PID controller for ABS system and vehicle dynamics model in MATLAB/Simulink and CarSim software, respectively. Section 5 verifies the improved expression through comparative analyses and discussions on various scenarios. The paper is concluded in Section 6.

\section{Wheel Dynamics and Optimal Slip Ratio}

The dynamic differential equations for the calculation of longitudinal motion of a vehicle are described as follows:

$$
\begin{aligned}
& m \dot{u}=-F_{x}, \\
& J \dot{\omega}=F_{x} R-T_{b}-T_{g}, \\
& F_{x i}=\mu_{i}(\lambda) F_{z i},
\end{aligned}
$$

where $i$ is $f l, f r, r l$, and $r r ; m$ and $u$ are a quarter of the vehicle mass and wheel velocity. $F_{x}$ is the driving resistance; $J$ is the wheel inertia; $\omega$ is the wheel rotational speed; $T_{b}$ and $T_{g}$ are the braking torque and the rolling resistance torque; $\mu_{i}$ and $F_{z i}$ are the friction coefficient and normal force of $i$ wheel, as shown in Figure 1.

During the braking process, the wheel speed $u$ can be larger than its rotation speed $R \omega$ which is characterised by the wheel longitudinal slip $\lambda$ :

$$
\lambda=\frac{u-R \omega}{u} .
$$

As shown in (3), the longitudinal force $F_{x}$ is described as a function that depends on the longitudinal friction coefficient $\mu(\lambda)$. If the longitudinal slip $\lambda$ is small, the relationship between the longitudinal force and slip is linear, but, with a further increase of the slip, the longitudinal force reaches the

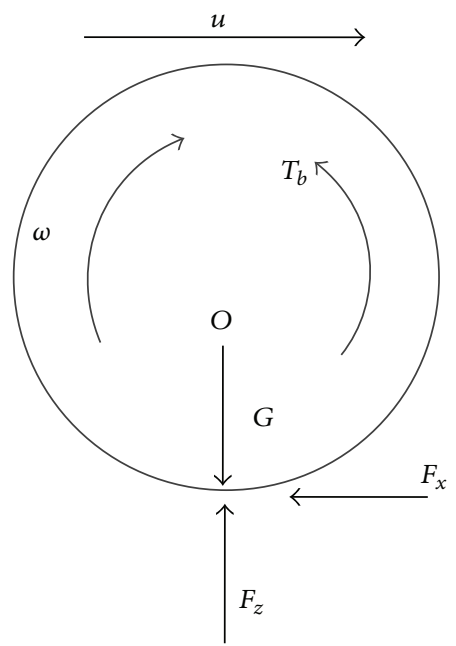

Figure 1: Single wheel model.

maximum at the certain value of the slip specified by tyreroad adhesion and is saturated beyond that. When the longitudinal force reaches a maximum value, the corresponding slip value is referred to as the optimal slip ratio.

In essence, the principle of ABS control is to always maintain the wheel slip ratio near the optimal slip ratio, which ensures a continuous maximum value onto tyre longitudinal force. In order to acquire the best braking effect, ABS schemes usually take the optimal slip ratio as the control target. Up to now, researchers have made numerous studies on the influence of road adhesion coefficient, vehicle velocity, and tyre slip angle changes on the optimal slip ratio [1-4].

Most studies about optimal slip ratio control focused on the real-time identification and estimation of unknown road condition, vehicle velocity, and tyre slip angle. Furthermore, based on a series of advanced control methods, ABS controller is optimized to keep the real wheel slip ratio closer to the theoretical optimal value. In principle, previous studies have been carried out mainly on the basis of the optimal slip ratio expression proposed by Liu [8] and Bian [9]:

$$
\lambda_{\text {op }}=\lambda_{0}+0.165 \times \log \left(\frac{64}{u}\right)+0.01 \times \delta^{1.5},
$$

where $\lambda_{\text {op }}$ is the optimal slip ratio, $\lambda_{0}$ is the road surface friction coefficient, $u$ is wheel velocity, and $\delta$ is the tyre slip angle. Equation (5) was developed on the basis of the tyre Magic Formula in the period from 1989 to 1994, excluding the influence of tyre inflation pressure changes. However, an increasing number of researches indicate that tyre inflation pressure change causes a direct influence on the relationship between tyre longitudinal force and slip ratio, which means that the value of optimal slip ratio could be influenced by tyre inflation pressures [6-8].

\section{Optimal Slip Ratio Prediction considering the Tyre Inflation Pressure}

In order to account for the influence of the tyre inflation pressure on the longitudinal friction characteristics, 


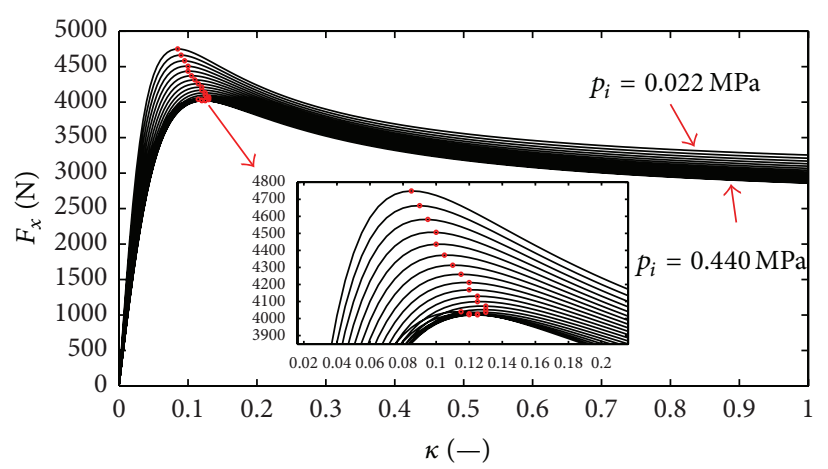

(a)

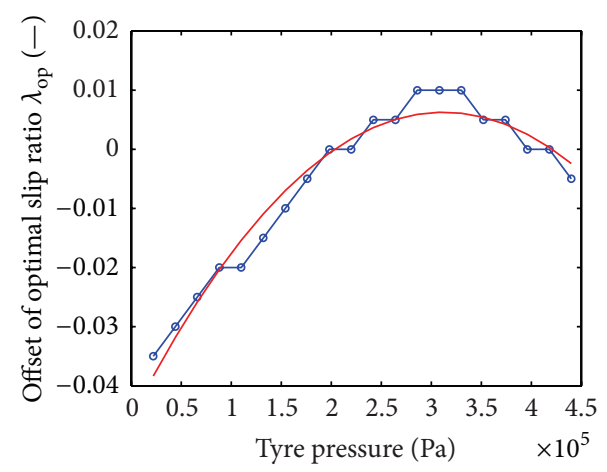

(b)

FIGURE 2: (a) $F_{x}-\lambda$ curves under different tyre inflation pressures. (b) Relationship between optimal slip ratio and tyre inflation pressures.

Besselink et al. [14] modified and improved the longitudinal tyre characteristic formula and related parameters. The main effects of tyre inflation pressure changes on the longitudinal tyre characteristics are identified as follows $[10,11]$ :

(i) Changes in longitudinal slip stiffness and camber stiffness.

(ii) Changes in longitudinal peak friction coefficient.

Therefore, the Magic Formula for predicting longitudinal force can be improved to

$$
\begin{aligned}
F_{x} & =\left(D _ { x } \operatorname { s i n } \left[C_{x}\right.\right. \\
& \left.\cdot \arctan \left\{B_{x} \lambda_{x}-E_{x}\left(B_{x} \lambda_{x}-\arctan \left(B_{x} \lambda_{x}\right)\right)\right\}\right] \\
& \left.+S_{V x}\right) G_{x \alpha},
\end{aligned}
$$

where $\lambda_{x}$ is the overall longitudinal slip; $B_{x}$ is the longitudinal stiffness factor; $C_{x}$ is shape factor for longitudinal force; $D_{x}$ is the peak value factor; $F_{z}$ is the vertical force; $E_{x}$ is the longitudinal curvature factor; $S_{v x}$ is vertical shift factor; and $G_{x \alpha}$ is comprehensive factor for combined slip, which are calculated by

$$
\begin{aligned}
D_{x} & =\mu_{x} F_{z}, \\
B_{x} & =\frac{K_{x \lambda}}{C_{x} D_{x}}, \\
K_{x \lambda} & =K_{x \lambda, \mathrm{nom}} \cdot\left(1+p_{p x 1} \cdot d p_{i}+p_{p x 1} \cdot d p_{i}^{2}\right), \\
\mu_{x} & =\mu_{x, \mathrm{nom}} \cdot\left(1+p_{p x 3} \cdot d p_{i}+p_{p x 4} \cdot d p_{i}^{2}\right),
\end{aligned}
$$

where $\mu, K, \mu_{x, \text { nom }}$, and $K_{x \lambda, \text { nom }}$ are longitudinal peak friction coefficient, longitudinal slip stiffness, and their corresponding nominal values, respectively. $p_{i}$ and $p_{i 0}$ are measured pressure and nominal tyre inflation pressure. And $d p_{i}=$ $\left(p_{i}-p_{i 0}\right) / p_{i 0}$ is dimensionless increment of tyre inflation pressure. As can be seen in both (9) and (10), there is an additional factor $\left(1+p_{p x i} \cdot d p_{i}+p_{p x(i+1)} \cdot d p_{i}^{2}\right)$ which is the product of the inflation pressure increment $d p_{i}$ and Magic Formula parameter $p_{p x i}$, highlighting the influences of pressure changes.
In order to investigate the quantitative influence of tyre inflation pressure changes on the optimal slip ratio, with tyre inflation pressure being considered as independent variable, relation curves of longitudinal force and slip ratio under different tyre inflation pressures, $F_{x}-\lambda$, are presented in Figure 2 based on the improved Magic Formula of (6). The peak longitudinal friction point of each curve is crucially marked and the set of optimal slip ratio points corresponding to the tyre pressure input is acquired. In the figure, the results were placed on a standard 205/60-R15 tyre under a vertical load of $5000 \mathrm{~N}$. To examine the influences of pressure changes, the tyre pressure changes from 0.1 times to 2 times of the nominal pressure, $0.22 \mathrm{MPa}$, obtaining twenty corresponding $F_{x}-\lambda$ curves as illustrated in Figure 2(a). As shown by the maximum longitudinal friction highlighted with the circle markers, the maximum longitudinal friction increases with decreasing in tyre pressure, whereas the optimal slip ratio decreases correspondingly. When the tyre inflation pressure is higher than the nominal value, the optimal slip ratio firstly increases and then decreases slightly. Fitting results show that the quadratic fitted curve correlates to the point set of optimal slip ratio very well with a correlation coefficient of more than 0.982 .

In order to take quantitative effect of inflation pressure into account, the optimal slip ratio in (5) can be modified by including two more terms as shown in the following equation:

$$
\begin{aligned}
\lambda_{\mathrm{op}}= & \lambda_{\mathrm{op}, \mathrm{nom}}+p_{p i 1} \cdot d p_{i}+p_{p i 2} \cdot d p_{i}^{2} \\
= & \lambda_{0}+0.165 \times \log \left(\frac{64}{u}\right)+0.01 \times \delta^{1.5}+p_{p i 1} \cdot d p_{i} \\
& +p_{p i 2} \cdot d p_{i}^{2},
\end{aligned}
$$

where $p_{p i 1}$ is the linear influence coefficient of tyre inflation pressure changes and $p_{p i 2}$ is the quadratic influence coefficient, both of which are decided by the characteristics of the tyre itself. The impact on the optimal slip ratio given by tyre inflation pressure changes has an extremely small coherence with other impact factors. Therefore, an independent secondorder factor should be essentially added into the optimal slip ratio prediction. 
TABLE 1: Simulation parameters.

\begin{tabular}{lccccc}
\hline$\lambda_{0}$ & 0.175 & Tyre type & $205 / 60-\mathrm{R} 15$ & $P_{p x 1} / P_{p x 2}$ & $-0.349 / 0.378$ \\
$v_{0 i} /(\mathrm{km} / \mathrm{h})$ & $100 / 80 / 60 / 40$ & Vehicle type & Sedan/C-class & $P_{p x 3} / P_{p x 4}$ & $-0.096 / 0.065$ \\
$\delta /^{\circ}$ & 0 & Engine power $/ \mathrm{kW}$ & 250 & $P_{p i 1}$ & -0.213 \\
$p_{i} / \mathrm{MPa}$ & $0.132 / 0.176 / 0.22 / 0.264 / 0.308$ & Max. braketorque $/ \mathrm{Nm}$ & 2000 & $P_{p i 2}$ & 0.179 \\
\hline
\end{tabular}

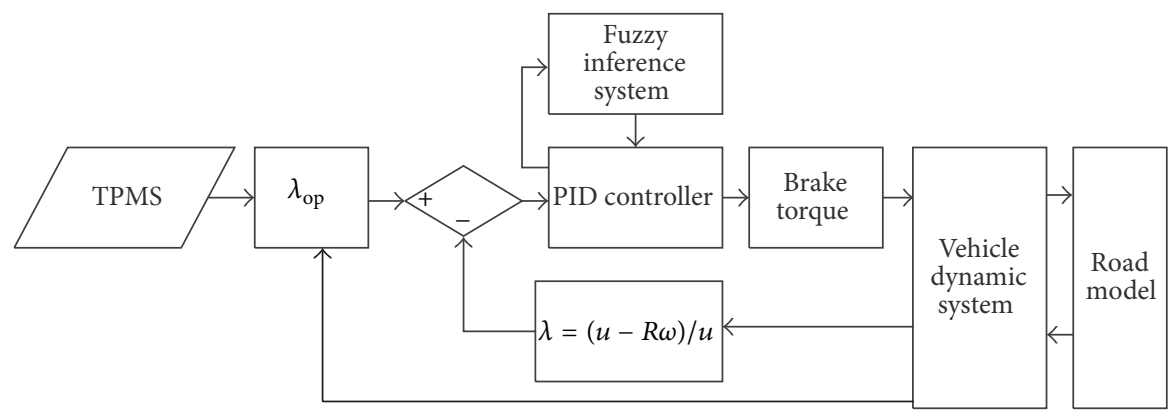

FIGURE 3: The block diagram of braking system based on the improved optimal slip ratio.

\section{ABS Controller Based on the Improved Optimal Slip Ratio}

The change of optimal slip ratio value can lead to a direct impact on the braking performance of ABS controller, which in turn changes both the braking distance and total braking time. In order to verify the improved expression and study the impact of tyre inflation pressure changes on braking performance, an ABS braking system based on the improved optimal slip ratio is designed, as shown in Figure 3. ABS braking system is composed of 4 parts: optimal slip ratio $\lambda_{\text {op }}$ estimator, slip ratio comparator, fuzzy PID controller, and execution system. There are 3 inputs for $\lambda_{\text {op }}$ estimator: tyre inflation pressure, vehicle velocity, and tyre slip angle. The road surface friction coefficient is commonly acquired by road type recognition algorithm. In order to simplify the computation, road surface friction coefficient is assigned to a constant value in this study. Real-time signal of tyre inflation pressure is acquired by direct measurement, such as commercially promoted Tyre Pressure Monitoring System (TPMS). The fuzzy PID controller is composed of a conventional PID controller and a fuzzy inference system.

Taking the error between actual slip rate and the optimal slip ratio $e$ and error rate $e c$ as inputs, fuzzy controller provides proportional coefficient $K_{p}$, integral coefficient $K_{i}$, and derivative coefficient $K_{d}$, its own output linguistic variables, as the input of PID controller. Design and selection of self-tuning principle, fuzzy control-rule table, membership functions, and universe range for the PID controller are referred to in $[12,13]$.

In order to comprehensively study the braking process and performance before and after the improvement of the optimal slip ratio, a cosimulation platform is established by combining the CarSim software and MATLAB/Simulink package based on a C-class passenger car, which then allow the simulation studies to be performed to evaluate the braking performance under different conditions. The time step is set to $0.01 \mathrm{~s}$ and Runge-Kutta ode 45 algorithm is used. Other key simulation parameters are shown in Table 1.

\section{Results and Discussion}

5.1. Influence of Tyre Inflation Pressure on Wheel Velocity and Displacement. Based on the cosimulation system, the braking process and performance of the vehicle are examined for the improved optimal slip ratios by comparing them with those of original ratios. The curves of vehicle braking displacement and wheel velocity under different tyre inflation pressures and optimal slip ratios are shown in Figure 4.

If $p_{i}=0.308 \mathrm{MPa}$, for example, the wheel velocity of ABS controller aims at improved slip ratio significantly lower than the curve of the original one, rather close to the curve of the nominal pressure throughout the second-half braking process as in Figure 4(a). In order to confirm that the change in wheel velocity is due to the improvement on ABS controller, which makes the wheel velocity continuously adapted to the current optimal slip ratio, it is necessary to conduct a contrastive study of real-time slip ratios controlled by ABS controllers aiming at original and improved optimal slip ratios. Next, the real-time tyre slip ratio curves under two optimal slip ratio expressions are presented in Figure 4(b). Theoretically, according to (11) and Table 1, when tyre inflation pressure is larger than nominal pressure, the value of optimal slip ratio considering tyre pressure will slightly increase compared with the traditional slip ratio. As is shown in Figure 4(b), ABS controller aims at improved optimal slip ratio to make the absolute value of the wheel slip ratio continuously greater than that of a traditional one, which is consistent with the theory. Therefore, the effectiveness of improved expression and ABS controller is proved.

The differences of original velocity minus improved velocity, $u_{\text {old }}-u_{\text {improved }}$, show that a deviation occurs from the speed under different tire pressure toward the speed corresponding to the nominal pressure, as is shown in Figure 4(c). 


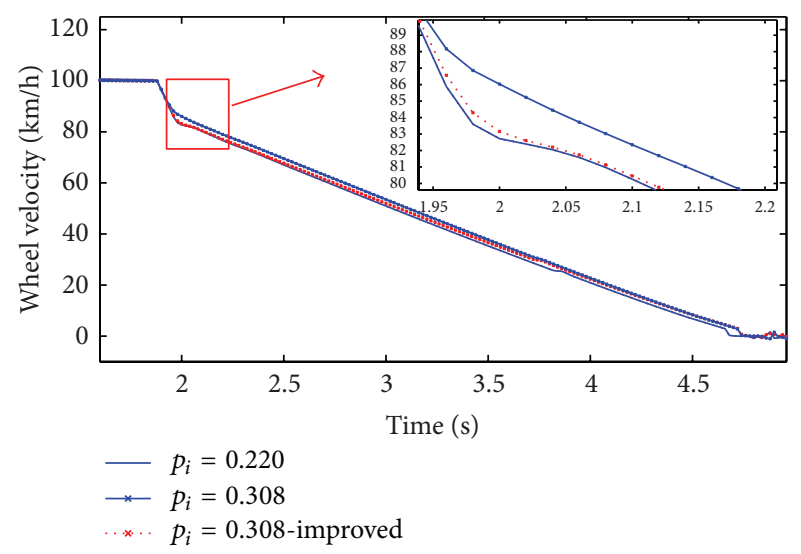

(a)

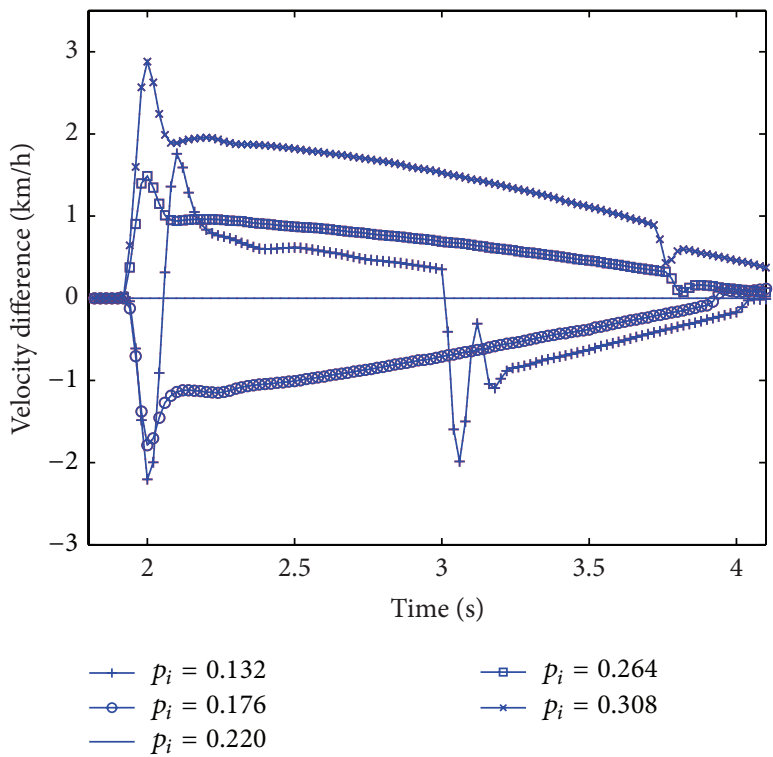

(c)

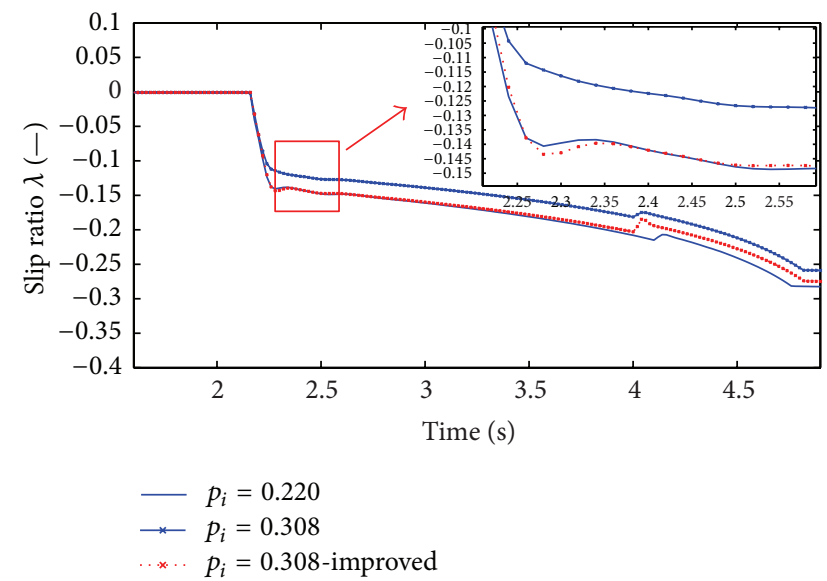

(b)

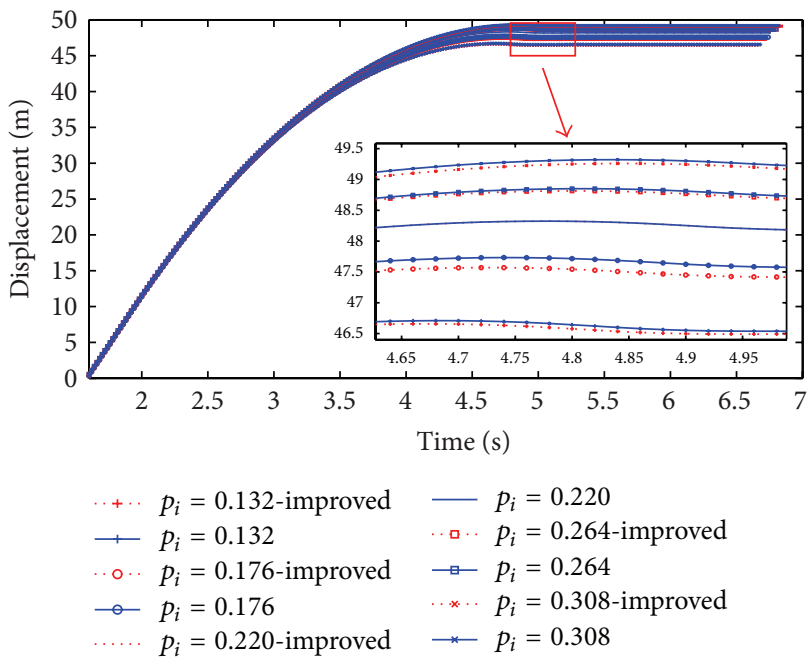

(d)

Figure 4: (a) Wheel velocities during the braking process. (b) Real-time tyre slip ratios during the braking process. (c) Differential value between the original velocity and the improved one. (d) Braking displacements under different tyre pressures and $\lambda_{\text {op }}$ predictions.

In particular, when tyre pressure is higher than the nominal value, the wheel velocity of improved slip ratio is consistently lower than that of original one during the braking process. That is why the velocity differences of $p_{i}=0.264 \mathrm{MPa}$ and $p_{i}=0.308 \mathrm{MPa}$ are always positive. When the pressure is lower than the nominal value, the wheel velocity curve shows the almost opposite trend. In addition, as to the low pressure case when $p_{i}=0.132 \mathrm{MPa}$, the wheel velocity in the inception phase fluctuates strongly and remains higher than the original wheel velocity during the steady phase, which proves that ABS controller has made timely amendments to the wheel speed based on the latest improved optimal slip ratio.

Compared with the original ABS controller, braking distances and braking time under all nonnominal tyre pressures become shorter, which proves that the improved ABS controller, aiming at the improved slip ratio, has optimized the wheel velocity and thus achieved the beneficial result as shown in Figure 4(d).

\subsection{Influence of Tyre Inflation Pressure on Braking Perfor-} mance. In order to investigate the impact of tyre inflation pressure changes on vehicle braking performance (braking distance and braking time), simulation studies were conducted under different initial braking speeds on the basis of the original slip ratio and improved optimal slip ratio.

As shown in Figure 5(a), both original braking distance and improved braking distance increase with the initial braking speed. In addition, there is a slight rising trend with increasing tyre pressure. The braking distances before and after improvement are very close. It is hard to distinguish the influence of the improved slip ratio on the braking distance. Likewise, variation trend of the braking time is similar to that 

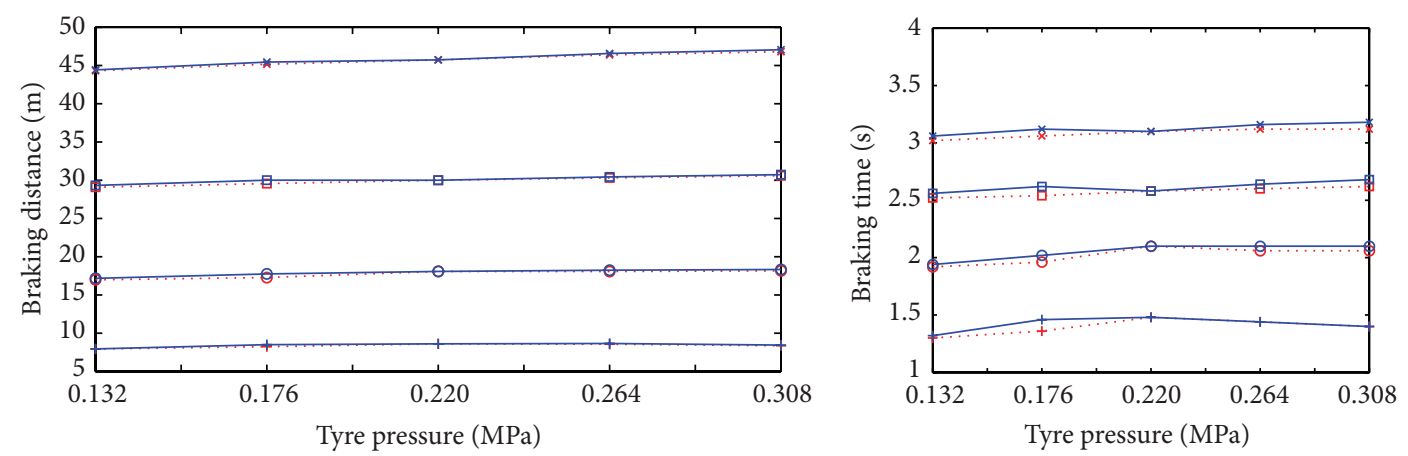

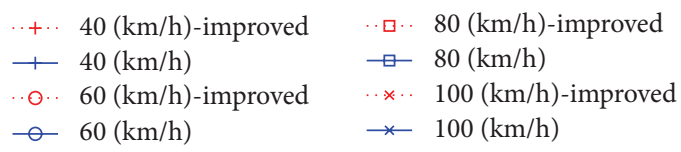

(a)

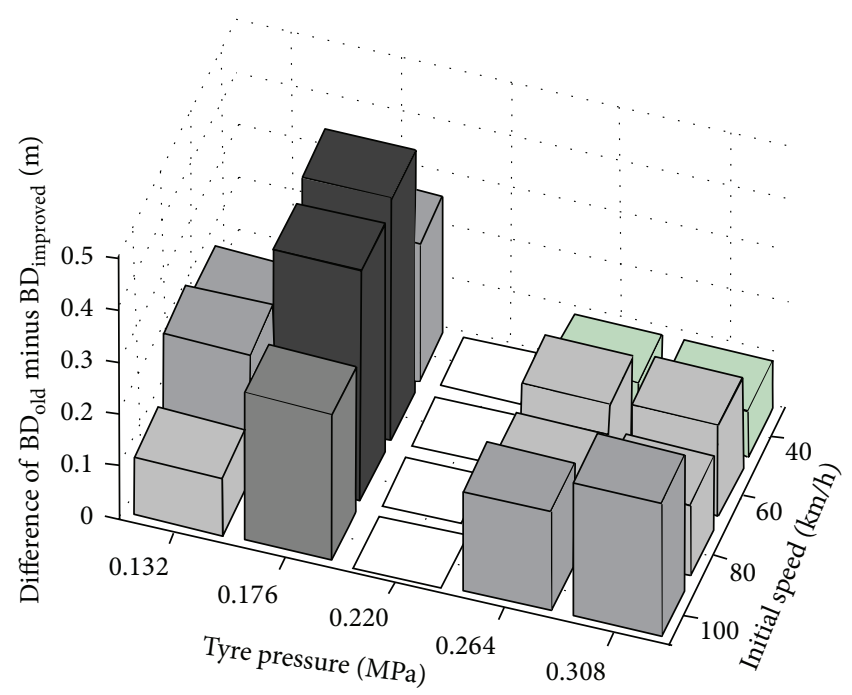

(c)

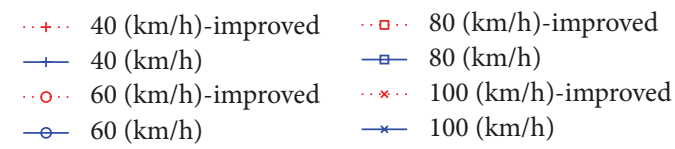

(b)

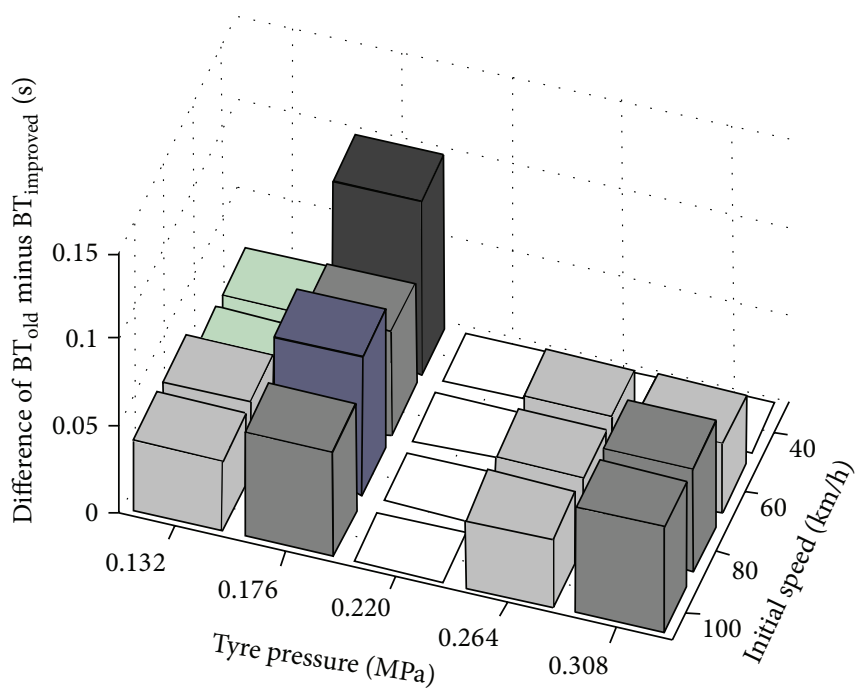

(d)

FIGURE 5: Braking performances under different initial braking speeds. (a) Comparison of braking distances before and after improvement. (b) Comparison of braking time before and after improvement. (c) Difference of original braking distance minus improved braking distance.

(d) Difference of original braking time minus improved braking time.

of the braking distance, and the difference of braking time is also hard to be identified.

In order to compare and analyze the degree of reduction, the difference of braking distance and braking time before and after improvement under each condition is calculated, respectively. As shown in Figures 5(c) and 5(d), under all tyre inflation pressures and initial braking speeds, the braking distance and braking time after the improvement are all effectively shortened. According to improved optimal slip ratio expression, the value of slip ratio decreases with lowering tyre pressure, and the corresponding longitudinal force $F_{x}$ increases. When tyre inflation pressure is higher than the nominal value, with the increasing pressure, the peak of $F_{x}-\kappa$ curve began to migrate to the right, accompanied by the shape deforming. That is, when the tyre inflation pressure is different from the nominal value, the corresponding longitudinal force of the optimal slip ratio, calculated by the original prediction which does not consider the tyre pressure changes, is not the real maximum longitudinal force. The corresponding longitudinal force predicted by the improved expression considering the tyre pressure is always more closer to the maximum braking force under the real-time tyre condition. It is obvious that the greater braking force is the shorter braking time will be. Therefore, the braking performance based on the improved optimal slip ratio has been enhanced. So it is in the case of tyre pressure lower than nominal pressure.

Based on the above analysis, the essence of ABS control, aiming at the improved optimal slip ratio, is by relocating the optimal slip ratio to keep real-time wheel slip ratio near the improved value and hence the transient longitudinal force $F_{x \text {,improved }}$ always in a maximum value under current tyre pressure. 
It can also be known from Figures 5(c) and 5(d) that when tyre inflation pressure is larger than nominal pressure differences of braking distance and braking time continuously increase with tyre pressure increasing. When tyre pressure is slightly lower than nominal pressure, optimization effect of the improved expression reaches the optimum. If tyre pressure continuously decreases, it tends to be less effective.

The relationship between performance differences and initial braking speed, as is shown in Figures 5(c) and 5(d), is not clearly or consistently related under the control of improved optimal slip ratio. This illustrates that the orthogonality between tyre pressure changes, wheel velocity, and other factors is good and introducing an independent second-order factor to characterize the influence of tyre inflation pressure on optimal slip ratio is reasonable and essential.

\section{Conclusions}

(1) Based on the ABS control aiming at improved optimal slip ratio, both vehicle braking distance and braking time, under all nonnominal tyre inflation pressures, are effectively shortened. It proves that ABS controller designed to the improved optimal slip ratio can fairly optimize the braking process under different tyre pressures.

(2) The difference of braking performances between original and improved optimal slip ratios does not change linearly with tyre inflation pressure changes. When tyre inflation pressure is slightly lower than the nominal value, the difference of braking performances is the most obvious.

(3) The influence of tyre inflation pressure changes on the prediction is less affected by initial braking speed of vehicle. Introducing an independent second-order factor to characterize the influence of tyre inflation pressure on optimal slip ratio is essential and rational.

\section{Notations}

$m: \quad$ A quarter of the vehicle mass

$u$ : Wheel velocity

$F_{x}$ : Longitudinal tyre force

$J$ : Wheel inertia

$\omega$ : Wheel rotational speed

$T_{b}$ : Braking torque

$T_{g}$ : Rolling resistance torque

$\mu_{i}$ : Friction coefficient

$F_{z i}$ : Normal force of $i$ th wheel

$R: \quad$ Wheel radius

$\lambda$ : Wheel longitudinal slip ratio

$\lambda_{\text {op }}$ : Optimal slip ratio

$\delta: \quad$ Tyre slip angle

$\lambda_{x}$ : Overall longitudinal slip

$B_{x}$ : Longitudinal stiffness factor

$C_{x}$ : Shape factor for longitudinal force

$D_{x}$ : Peak value factor

$E_{x}$ : Longitudinal curvature factor
$S_{v x}:$ Vertical shift factor

$G_{x \alpha}$ : Combined slip factor

$K: \quad$ Longitudinal slip stiffness

$p_{i}$ : Tyre inflation pressure

$p_{i 0}$ : Nominal tyre inflation pressure

$d p_{i}$ : Dimensionless increment of tyre inflation pressure

$p_{p x i}:$ Magic Formula parameter

$p_{p i 1}$ : Linear influence coefficient of tyre inflation pressure changes

$p_{p i 2}$ : Quadratic influence coefficient.

\section{Conflict of Interests}

The authors declare that there is no conflict of interests regarding the publication of this paper.

\section{Acknowledgments}

The support of the High-Tech Industrialization Projects of Shanxi Province, China (no. 2011-2368) and The Natural Science Foundation of Shanxi Province, China (no. 20120110242 ) is gratefully acknowledged. Thanks are due to Dan Song of State Grid Skill Training Centre of SEPC for her help in ABS controller modelling. The authors acknowledge the valuable comments and discussions with Hangbin Zhu of TASS International and Yonghong Wang of Shanxi Dayun Automobile Manufacturing Co., Ltd.

\section{References}

[1] G. F. Mauer, "A fuzzy logic controller for an ABS braking system," IEEE Transactions on Fuzzy Systems, vol. 3, no. 4, pp. 381388, 1995.

[2] H. Wang and L. Yang, "Simulation of automotive ABS using fuzzy self-tuning PID control," Journal of Transportation Systems Engineering and Information Technology, vol. 12, no. 5, pp. 52-56, 2012.

[3] W. Zhu and Y. Chen, "Application and simulation of automotive ABS using fuzzy PID control," Journal of Jiangsu University (National Science Edition), vol. 25, no. 4, pp. 310-314, 2004.

[4] V. Ćirović, D. Aleksendrić, and D. Mladenović, "Braking torque control using recurrent neural networks," Proceedings of the Institution of Mechanical Engineers, Part D: Journal of Automobile Engineering, vol. 226, no. 6, pp. 754-766, 2012.

[5] H. Guan, B. Wang, P. Lu, and L. Xu, "Identification of maximum road friction coefficient and optimal slip ratio based on road type recognition," Chinese Journal of Mechanical Engineering, vol. 27, no. 5, pp. 1018-1026, 2014.

[6] B. Wang, "Simulation of automobile ABS based on online tracking varying optimal slip," Journal of Hubei Automotive Industries Institute, no. 1, pp. 5-9, 2011.

[7] M. Mirzaei and H. Mirzaeinejad, "Optimal design of a nonlinear controller for anti-lock braking system," Transportation Research Part C: Emerging Technologies, vol. 24, pp. 19-35, 2012.

[8] Z. Liu, "Mathematical models of tire-longitudinal road adhesion and their use in the study of road vehicle dynamics," Journal of Beijing Institute of Technology, no. 2, pp. 193-204, 1996. 
[9] M. Bian, "Simplified tire model for longitudinal road friction estimation," Journal of Chongqing University of Technology (Natural Science), vol. 26, no. 1, pp. 1-5, 2012.

[10] H. Pacejka, Tire and Vehicle Dynamics, Elsevier, 2005.

[11] M. Massaro, V. Cossalter, and G. Cusimano, "The effect of the inflation pressure on the tyre properties and the motorcycle stability," Proceedings of the Institution of Mechanical Engineers D: Journal of Automobile Engineering, vol. 227, no. 10, pp. 14801488, 2013.

[12] K. Parczewski, "Effect of tyre inflation preassure on the vehicle dynamics during braking manouvre," Eksploatacja i Niezawodnosc, vol. 15, no. 2, pp. 134-139, 2013.

[13] H. Taghavifar and A. Mardani, "Investigating the effect of velocity, inflation pressure, and vertical load on rolling resistance of a radial ply tire," Journal of Terramechanics, vol. 50, no. 2, pp. 99-106, 2013.

[14] I. J. M. Besselink, A. J. C. Schmeitz, and H. B. Pacejka, "An improved Magic Formula/Swift tyre model that can handle inflation pressure changes," Vehicle System Dynamics, vol. 48, supplement 1, pp. 337-352, 2010. 

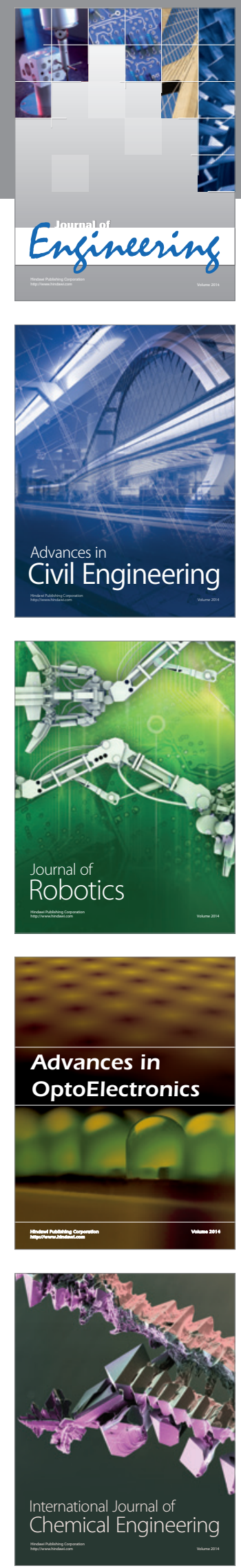

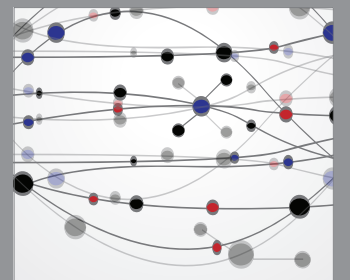

The Scientific World Journal
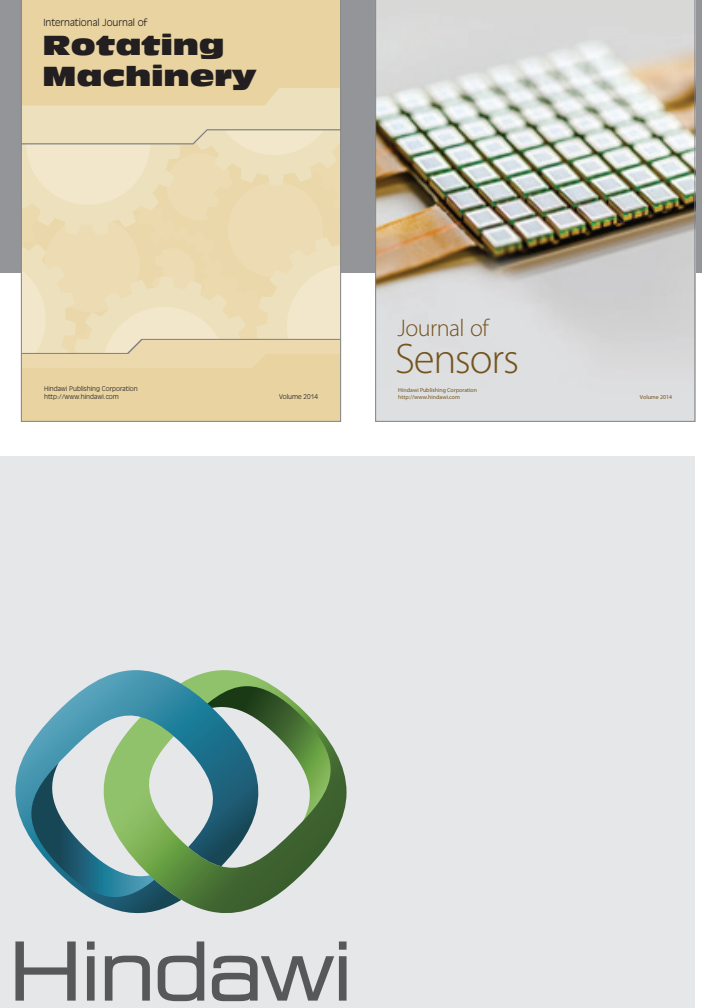

Submit your manuscripts at http://www.hindawi.com
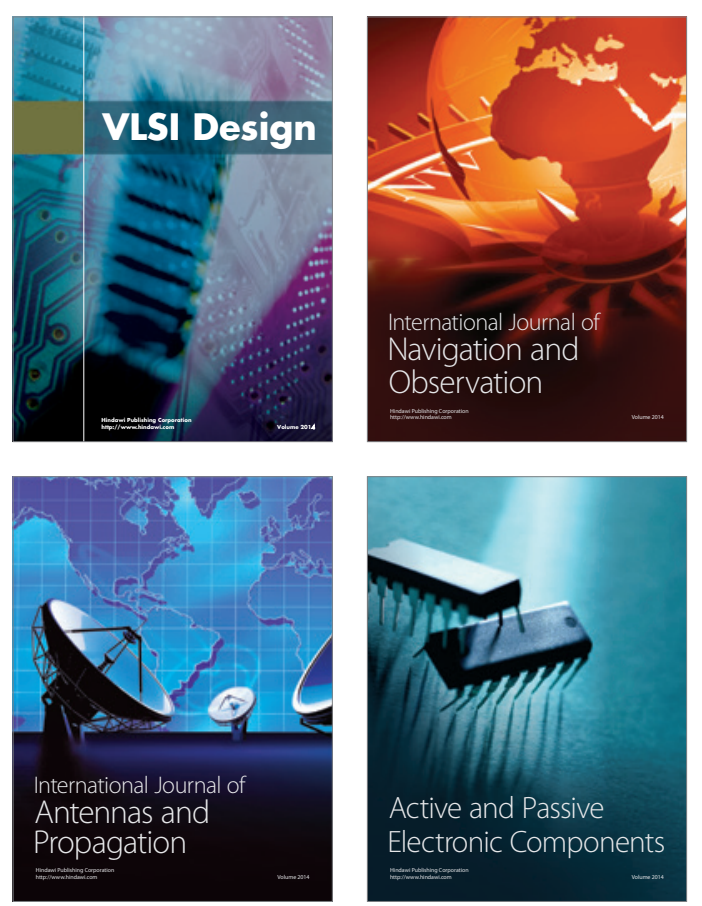
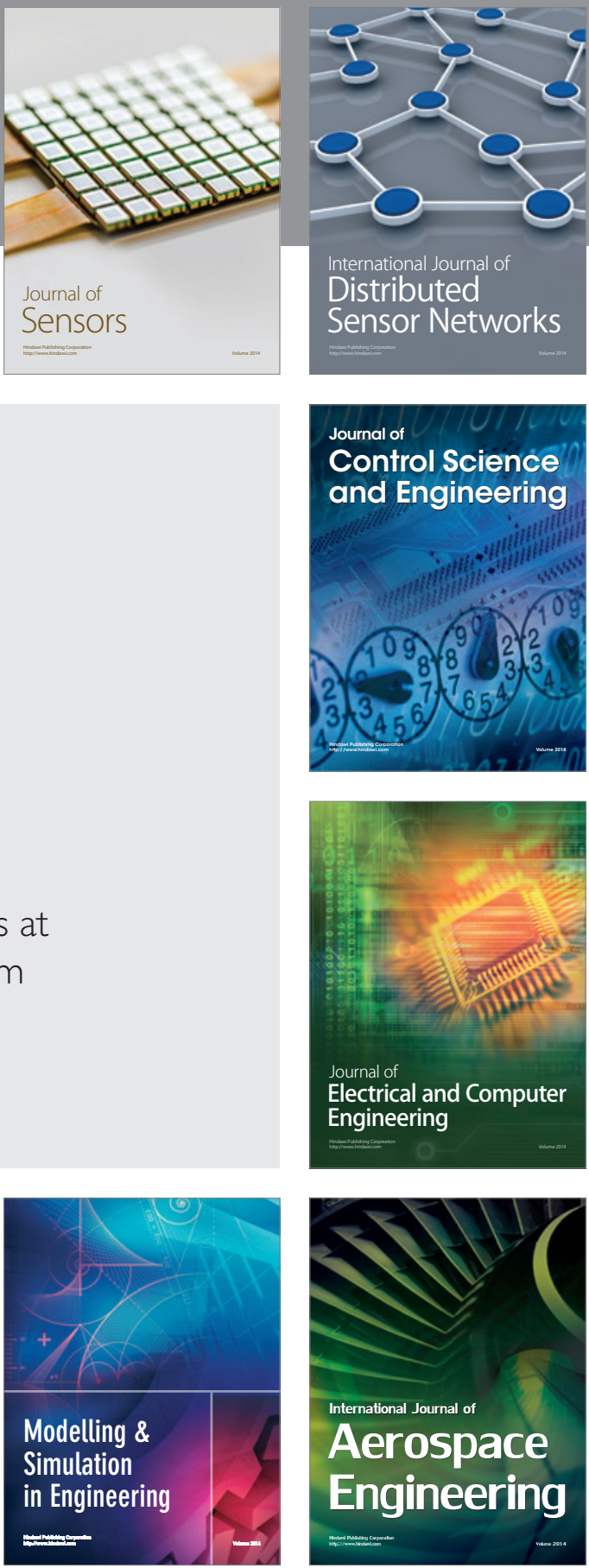

Journal of

Control Science

and Engineering
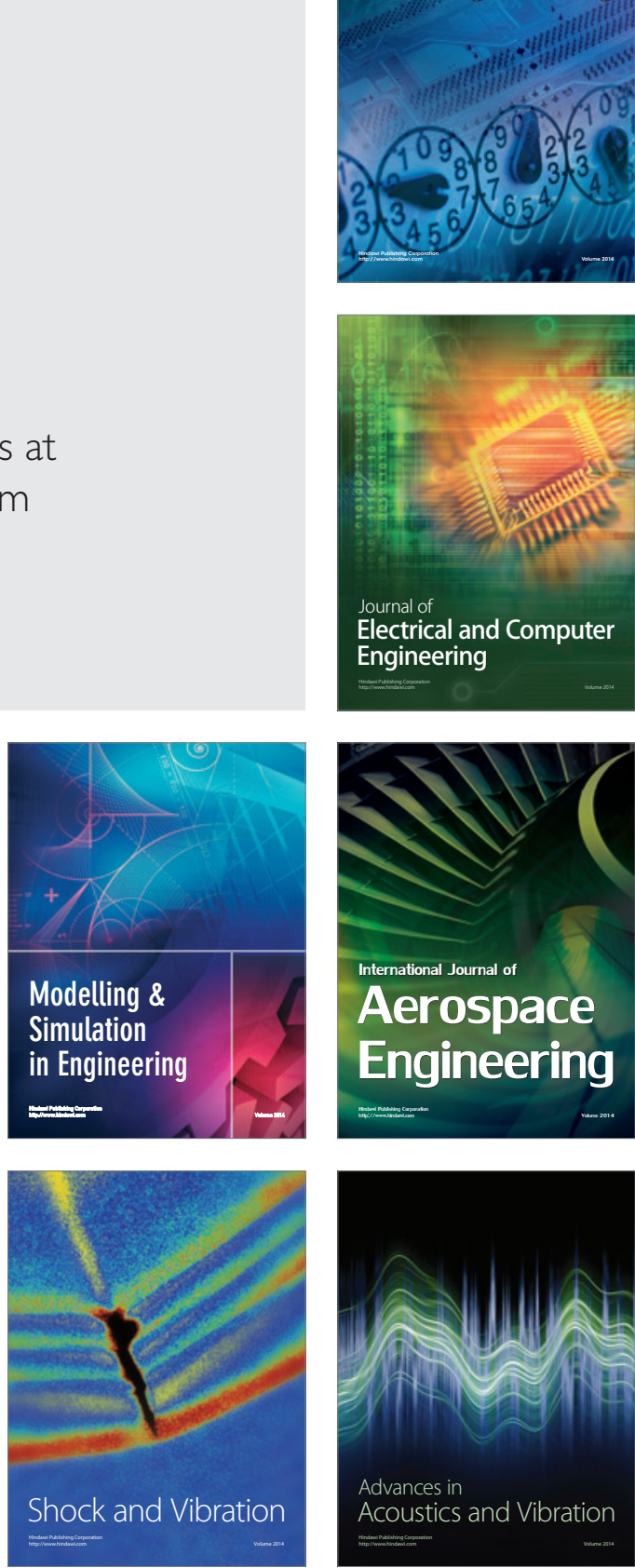